\title{
LA RELACIÓN Y SU FUNCIÓN COMO MODELADORA DE LA
}

IMAGEN

\author{
Dr. Maximiliano Ortner ${ }^{1}$ \\ Universidad Nacional de La Matanza \\ max@ortnercom.com.ar
}

Material original autorizado para su primera publicación en la revista académica REDMARKA. Revista Digital de Marketing Aplicado

https://doi.org/10.17979/redma.2011.01.07.4726

Recibido: 16 Agosto 2011

Aceptado: 2 Diciembre 2011

\section{Resumen}

Comprender las relaciones propone un desafío singular a la hora de presentar nuevas ideas o conceptos a los públicos de una organización. De las propuestas relaciones resultará el éxito en desarrollar una relación que será la génesis de una imagen positiva acerca de la organización y sus productos o servicios en la mente de los públicos, ya sean estos clientes, usuarios o bien relacionados desde la comunicación.

Desde un modelo gráfico donde se analiza la emisión y la recepción de los mensajes de acuerdo al número de públicos e interlocutores se podrá comprender el flujo y el alcance de la información que busca convertirse en imagen.Esta idea de especialización estratégica en las relaciones redundará en asignarle el valor correcto a los intereses, necesidades y expectativas de los públicos.

\footnotetext{
${ }^{1}$ Doctor en Ciencias de la Comunicación Universidad del Salvador. Master en Dirección de Comunicaciones Institucionales UCES. Postgrado de Desarrollo de Negocios IAE. Licenciado en Marketing. Su tesis doctoral trata la construcción de las relaciones entre las organizaciones y sus públicos. A cargo de las cátedras de Imagen y Comunicación Institucional, y Marketing en la Universidad Nacional de La Matanza (UNLaM)

Dirige una organización de asesoramiento e investigación en temas de comunicación organizacional. Ha ocupado puestos ejecutivos en diversas organizaciones en las áreas de marketing y de relaciones institucionales.
}

REDMARKA UIMA-Universidad de A Coruña - CIECID 
Palabras clave: Relaciones, imagen, posicionamiento.

\section{Abstract}

The correct understanding of relationships constitutes a challenge between the organization and its audiences when it comes to introduce new ideas or concepts. These proposals for a relationship will sum up in building animage about the organization, its products and services in the publics' mind, whether they are customers, users or other communication related audiences.

In order to understand the range, flow and reach of the information before it becomes an image, we will analyze by means of a graphic model the way messages emitted and received according to the number of audiences and parties involved in the process. This strategic approach will serve as a mean to assign proper value to the audiences' interests, needs and expectations.

Keywords: Relationships, image, positioning. 


\section{Introducción}

La construcción de las relaciones desde el ambiente interno de la organización supone una definición estratégica para establecer un contacto bidireccional entre el público o los públicos seleccionados y la institución. Para poder determinar el valor que tienen las relaciones para los públicos será necesario identificar algunos conceptos provenientes de las ciencias biológicas con el fin de pasar, luego, a las disciplinas relacionadas con la comunicación, como pueden ser las Relaciones Públicas, las Comunicaciones Corporativas y el Marketing. Comenzando por las biológicas se encuentra el caso de la teoría de la Autopoiesis.

En su descripción de la Autopoiesis, Humberto Maturana y Francisco Varela, explican cómo funciona esta fuerza que impulsa a los organismos a subsistir (Maturana y Varela, 2003, 99). Como una fuerza o pulsión de vida (en términos freudianos), requiere de relaciones entre distintos seres para asegurar su supervivencia. Esta teoría sobre la evolución de los organismos se puede aplicar directamente al campo de la comunicación a través de un experimento llevado a cabo por el Dr. John Conway llamado el Juego de la Vida².

En este juego se muestra el valor de las relaciones, observándose mediante la asociación de un organismo con otro el desarrollo conjunto obtenido mediante la suma de valor, hasta la creación de nuevos organismos emergentes. Del mismo modo, Richard Dawkins en "El gen egoísta", toma la idea desde la teoría de la evolución de las especies, identificando el gen como la partícula mínima de transmisión para definir cómo mediante las relaciones y la asociación pueden adaptarse y así perpetuar su existencia. Mientras que sin las relaciones terminarían por extinguirse.(Dawkins, 1993, 27)

\footnotetext{
${ }^{2}$ TheGame of Life es una simulación matemática basada en reglas determinadas creado por el Dr. John Conway que representa el ciclo de vida de un organismo o un conjunto de ellos. A partir de sus asociaciones con otros organismos se puede determinar su reproducción, su muerte o su subsistencia.
} 
En un paralelismo directo con las relaciones humanas y organizacionales, los vínculos desarrollados en los que existe una comunicación continua y bidireccional favorecen el crecimiento mutuo cooperativo por el aporte y la suma de valor individual. Una propuesta de valor funciona en una relación como la del niño que lleva la pelota a un grupo de amigos. La pelota en este caso es la propuesta que crea valor, que transforma un grupo en dos equipos y que organiza la relación entre ambos en un partido de fútbol.

En el mundo corporativo, la propuesta de valor tradicionalmente fue entendida como el producto, que obra como vínculo entre el comprador o usuario y la empresa que lo produce. En este enfoque sólo se toma en cuenta el público o los públicos que representan el target comercial de la empresa o institución, dejando de lado al resto de los públicos que interactúan con ella.

No obstante, los públicos no funcionan como compartimientos estancos y los integrantes de un público no se autoexcluyen de participar en el conjunto. Esto hace posible encontrar clientes y consumidores con un fuerte interés por cómo la organización se comporta, por ejemplo, respecto al medioambiente o a la comunidad, o que incluso pueden estar decididos a tomar acciones consecuentes contra una empresa que no lo hace correctamente o que no demuestra un compromiso detrás de sus mensajes, aun a pesar de haber consumido sus productos hasta el momento. 


\section{La construcción de la relación}

El hecho de "construir" una relación significa que se está realizando un esfuerzo consciente por desarrollar lazos con ciertos públicos. Para lograrlo se deben establecer propuestas de valor que cumplan con el conjunto de sus expectativas acerca de la organización (ver ilustración). Si bien las expectativas pueden estar relacionadas con las de los públicos sobre la organización como un todo (Scheinsohn, 2000, 129-130), las concernientes al momento del relacionamiento responden también a los intereses particulares de cada individuo que forma parte del público.

En este caso, surge la idea de los intereses en paralelo a la de las expectativas. Los intereses estarán más centrados dentro del ámbito de las preocupaciones personales, mientras que el conjunto de expectativas será dictado por el público como un grupo de personas englobadas a través de uno o varios identificadores en común.

Sin embargo, cuando se debe pensar en grandes números de personas y en diversos públicos, la construcción de las relaciones es una manera efectiva de formalizar un intercambio ininterrumpido de información con los mismos a través de las relaciones de hecho. Es decir, generar una transferencia simbólica entre los públicos y la organización de modo que se creen vínculos que la formalicen y definan las razones de la unión relacional.

Este tipo de relación incluye las relaciones estratégicas construidas a través de acciones de publicidad, relaciones públicas $u$ otras herramientas de la comunicación que tienen la cualidad de generar lazos más profundos que están al nivel de los que construyen la imagen (ver ilustración). Así, se genera un una 
simbiosis entre relación e imagen, que en conjunto se potencian para definir en el tiempo una reputación.

\section{Componentes de la Reputación}

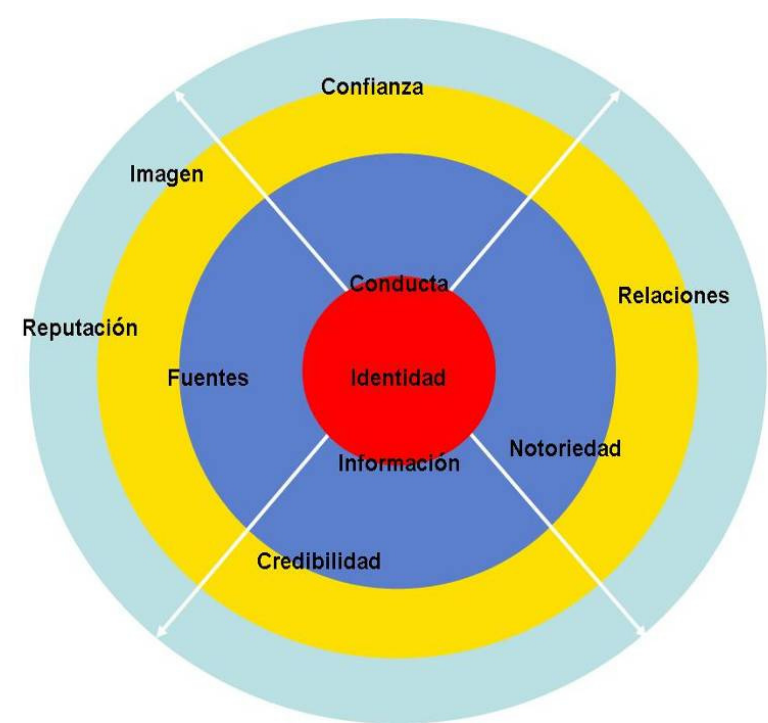

(Fuente: elaboración propia)

Las percepciones de los públicos en todos los aspectos de la comunicación de la organización, desde lo meramente visual hasta lo abstracto e incluso lo proveniente del exterior de la organización, harán de la relación una factibilidad. Asimismo, lo harán con la imagen.

Charles Fombrun describe cómo las relaciones se proponen a partir de lo simbólico (Fombrun, 1996,142). Éstas pueden influenciar positivamente el juicio que las personas forman acerca de la organización. Los vehículos para la transferencia de lo simbólico pueden ser:

- Los medios de comunicación, que emiten publicidad y en los que los periodistas informan en favor de las actividades de la empresa.

- Los analistas de inversión que asesoran a los posibles capitalistas de las compañías. 
- El boca a boca a través de la red informal por donde se propaga información, rumores e insinuaciones acerca de las instituciones y sus ejecutivos.

Sin embargo, es necesario entender las posibilidades de distribución de la información desde el emisor o los emisores hacia uno o más públicos.

De este modo se puede esquematizar esta noción a partir de cuatro cuadrantes representando cuatro posibles escenarios que permiten explicar las condiciones de distribución de la información, según el modo cómo los públicos, en su función de receptores, reciben la información de uno o muchos emisores.

\section{Representación de la matriz de distribución de la información entre emisores} y receptores

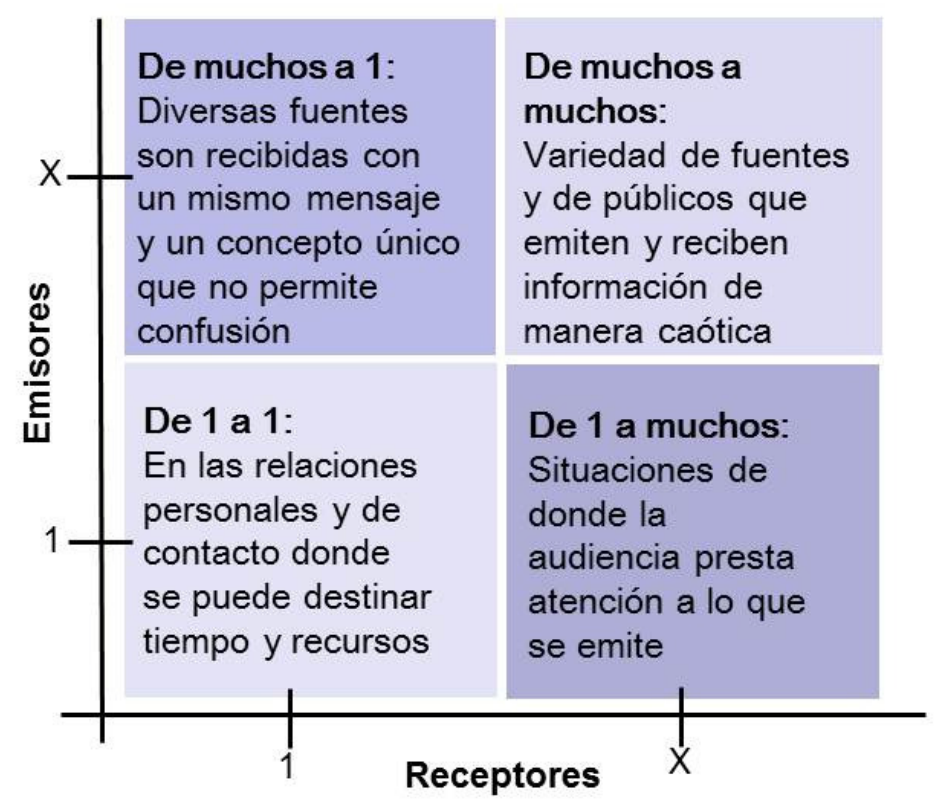

(Fuente: elaboración propia)

La distribución de los escenarios se divide de la siguiente manera: 
- De uno a uno: Situaciones donde la comunicación es directa. En el entorno relacional este caso se da especialmente cuando se trata de relaciones de tipo personal o de contacto

- De uno a muchos: Sin importar el tipo de relación que se mantenga con el o los públicos presentes, se cuenta con la atención de éstos. Éste puede ser el caso de una presentación o bien de una aparición en los medios a través de un periodista o presentador que acapare la audiencia.

- De muchos a uno: Diversas fuentes de información destinadas a un mismo público. En el caso de responder a una misma estrategia de comunicación se puede presentar un caso de éxito, ya que se trataría de una intervención fructífera sobre la imagen que posee ese público determinado.

- De muchos a muchos: El menos conveniente de los escenarios y sin embargo el más frecuente, donde conviven una multiplicidad de públicos y de emisores de mensajes. Los emisores pueden presentar ofertas de información acerca de la misma persona u organización pero proveyendo contenidos opuestos. Se trata de una situación compleja y caótica que escapa al control de la organización.

En este terreno conviene citar a Ivy Lee, uno de los pioneros de las Relaciones Públicas en la biografía de la familia Rockefeller: "...el éxito para tratar con las masas, reside en el arte de lograr ser creído." (Horowitz, 1976, 152) Pero para lograr este cometido, primero es necesario construir un puente firme entre los públicos y la organización. Es en ese momento que será posible ser creído y por supuesto aplicar los conceptos de Fombrun.

En otra nota acerca de las Relaciones Públicas, el entorno y la construcción relacional parecen una situación dada a partir del nombre de esta disciplina. Sin embargo, todavía no se han encontrado métodos propuestos desde los claustros académicos que sean aceptados y adoptados por los profesionales del área. En 
un paper presentado por Trent Seltzer ${ }^{3}$ de la Universidad de Florida al Institute for Public Relations, se define que: “[...] todos los estudios sugieren puntos importantes para el uso de las relaciones como una unidad de análisis en las Relaciones Públicas:

1. La calidad de la relación puede ser vista como una construcción independiente de los sistemas de medición comúnmente utilizados por las relaciones públicas para medir cambios de actitud y conducta.

2. El concepto de relación se encuentra basado en gran medida sobre percepciones compartidas de ambas partes.

3. Las relaciones cambian con el paso del tiempo.

4. Las relaciones pueden ser medidas." (Seltzer, 2005)

Así, puede parecer al observador que la función de estas disciplinas es la de crear, desarrollar y mantener relaciones, y probablemente es a lo que se aspire. No obstante, los profesionales se han limitado a desarrollar un juego de influencia y manipulación para lograr modificar las opiniones y la conducta de los públicos sin trabajar en los componentes relacionales como la confianza, la comunicación dialógica, el entendimiento de las necesidades y las percepciones, la empatía, etcétera.

El Dr. Federico Rey Lennon, autor de un libro sobre el padre de las Relaciones Públicas, Edward Bernays, cita a este último diciendo: “...Señalé la función social de las Relaciones Públicas en combatir el pensamiento estereotipado que impulsa al público a oponerse a los nuevos puntos de vista..." (Rey Lennon, 2006, 79) Si bien se destaca en esta publicación que E. Bernays era un firme estudioso de la psicología y de los aspectos relacionales de las relaciones públicas, el devenir de los años ha hecho que los especialistas centren su enfoque en los medios de comunicación como vía regia de alcance para sus públicos en lugar de desarrollar

\footnotetext{
${ }^{3}$ Selzer, Trent. "Measuring the Impact of Public Relations: Using a Coorientational Approach to Analyze the Organization-Public relationship" Submitted to the Institute for Public Relations for the 2005 Ketchum Excellence in Public Relations Research Award. [Los destacados en negrita se encuentran en el original.]
} 
un trabajo más profundo basado en el conocimiento de los mismos y en su conducta.

Al tomar el tema del diálogo, surge una postura que trabaja este concepto desde lo interpersonal, dejando a los profesionales del área en una situación difusa dado el bajo nivel de conocimiento que se ostenta para poder poner en funcionamiento un diálogo fluido entre una organización y sus públicos. Así lo exponen M. L. Kent y M. Taylor en su presentación académica publicada en 2002: "A pesar de que un enfoque dialógico en las relaciones públicas no es fácil de operar o al menos, es pasible de ser reducido a una serie de pasos, el diálogo consiste en distintas asunciones coherentes. La literatura sobre relaciones de las relaciones públicas, la comunicación, la filosofía y la psicología, revelan que existen cinco puntos dogmáticos del diálogo.

...[Estos son] mutualismo como el reconocimiento de la relación organizaciónpúblico; colaboración en los encuentros espontáneos con los públicos; empatía para sustentar y confirmar los intereses y objetivos de los públicos; riesgo como la voluntad de interactuar con los públicos bajo sus propios términos y por último, compromiso de darse al diálogo y a comprender las causas del comportamiento y las opiniones de los públicos." (Kent y Taylor, 2002, 25)

Para hablar de relaciones no es necesario sumar un conjunto de acciones sino tomar los pasos necesarios, como los descritos por Kent y Taylor, donde la organización se vuelque a un nuevo modelo mental que le permita presentarse como un actor transparente que no está tratando de influenciar sino que pretende desarrollarse en conjunto con la sociedad y no a partir de ella.

\section{De las relaciones de uno a la relación de muchos}

Cuando se trata de las relaciones entre empresa y públicos, es necesario no perder de vista que las relaciones se basan en la premisa de que son de persona 
a persona. En el caso de las relaciones con grupos, se produce el mismo caso pero individualmente se refiere a integrantes.

Aquí entran en juego los aspectos psicológicos presentados anteriormente. Los juegos de la psicología transaccional, los esquemas de necesidades de Maslow, la Escalera de la Inferencia y el espejo de Lacan. Cada uno de estos conceptos y teorías funcionarán en conjunto para determinar cada relación.

Cada persona determinará el tipo de relación que tendrá con la organización y así se desarrollará personalmente. Para que esta relación prospere debe estar basada en la piedra fundamental de toda relación: la confianza. En palabras de Gerard Apfeldofer, se debe dar y recibir (Apfeldorfer, 2005, 35). O bien, mejor expresado a través del Doctor Marcelo Manucci, la relación crecerá cuando ambas partes en su intersección generen una propuesta de valor mutua.

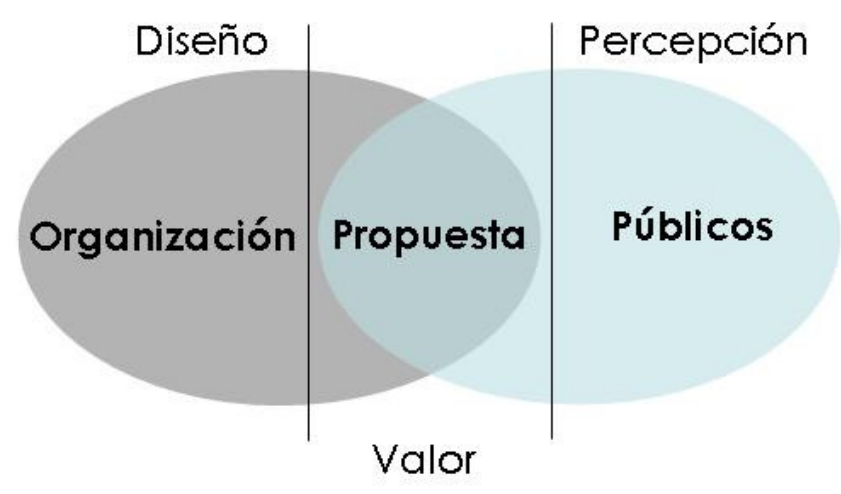

Cada propuesta de comunicación que se presenta en el día a día de una organización es, en definitiva, una propuesta de relación y supone una negociación. El proceso de negociación se superpone continuamente a la comunicación dado que se trata de un intercambio entre partes que pueden tener intereses y objetivos tanto similares como totalmente dispares. En este proceso, las partes buscan alternativas de llegar a un acuerdo que les proporcione resultados satisfactorios. De este modo habrá resoluciones que dejarán satisfecha 
a una parte y no a la otra, o viceversa, que no servirán para llegar a acuerdos; y sólo las que dejen a ambas partes satisfechas con el fruto del intercambio permitirán iniciar un camino de relacionamiento progresivo.

Las conexiones entre las técnicas de negociación y las relaciones suponen amplias zonas de contacto a partir del esquema del proceso de conformación de las relaciones presentado en páginas anteriores dado que en la etapa de "anclaje de la relación" tiene lugar una negociación donde se contraponen las propuestas de ambas partes buscando cubrir las expectativas o bien llegar a una relación que pueda prolongarse en el tiempo.

Helena Cornelius propone alternativas para enfrentar problemas relacionales a través de un enfoque de beneficio mutuo (Cornelius, 1995, 22-23). El enfoque presentado posibilita, de manera práctica, la implementación de las propuestas de Manucci y Apfeldorfer mediante la comprensión de cada situación y en el reconocer que cada participante de los públicos es una persona que debe ser reconocida como tal y bajo ninguna condición puede ser subestimada.

Como se describió ad supra, los desenlaces en la negociación suponen cuatro escenarios posibles en la relación entre la organización y sus públicos. Manucci, desde la postura de la propuesta de valor, explica que los acuerdos que inician una relación en una propuesta que no es conveniente para ambas partes serán vistos como una relación forzada y tendrán una tendencia a romperse. (Manucci, 2006, 122)

Roger Fisher y William Ury explican que en el proceso relacional que ocurre durante la negociación afloran aspectos a considerar. Los procesos psicológicos, emocionales y conductuales serán los condicionantes para determinar el resultado de la negociación. Algunos de los issues descritos son (Fisher y Ury, 1991, 158):

- Balanceo entre lo emocional y racional 
- $\quad$ Fluidez del intercambio comunicacional

- $\quad$ Grado de confianza

- $\quad$ Actitud hacia la aceptación o el rechazo

- Énfasis y reacción relativos hacia la persuasión o la coerción

- $\quad$ Grado de entendimiento mutuo

Estos puntos resumen los aspectos internos de los participantes de la negociación que se desarrolla en el momento del anclaje de la relación. Tener estos aspectos en cuenta hace imprescindible no sólo conocer de la mejor manera posible al público con el que la organización entabla negociación, sino que deberá interiorizar estos issues en el proceso de determinación del mensaje y principalmente en la propuesta de valor que se llevará a la mesa de negociación figurada que ocurre continuamente en el entorno de las comunicaciones institucionales.

Éste es uno de los mayores escollos para las organizaciones a la hora de interactuar con una persona en particular. Por lo general, las instituciones se encuentran preparadas para atender a las quejas de sus consumidores o usuarios desde la perspectiva comercial. Sin embargo, no lo están para atender sus frustraciones respecto de la compañía como un todo o si el problema es en referencia a su proceder con la comunidad, el medio ambiente u otro aspecto social.

Es en estos puntos donde las organizaciones suelen fallar, dejando flancos débiles expuestos que redundan en fallas de contención y finalmente, en la carencia de una propuesta de valor a nivel personal. Sin bien esta situación no responde por todo el trabajo de comunicación y relaciones que realiza la institución, no quita que a partir de esto pueda desencadenarse un malestar entre otros participantes de los públicos que hayan entrado en contacto. 
El término inglés empowerment surge como una propuesta posible de solución a este tipo de problema. JanCarlzon (Carlzon, 1991, 94) descubrió, en su paso como ejecutivo de primera línea en diversas líneas aéreas escandinavas, que los pasajeros no obtenían respuestas más allá de lo mínimo necesario ante demoras u otros inconvenientes de viaje. Su solución consistió en integrar a toda la organización a través de una estrategia y así, permitirles tomar sus propias decisiones de acuerdo a esta idea madre que había acordado. De este modo se eliminaron los flancos débiles. Asimismo, cada interlocutor estaba al tanto de la propuesta de valor que la compañía tenía y estaban listos para atender a cualquier interlocutor que lo requiriera sin miedo a enfrentar consecuencias por hacer más de lo que debían según su descripción de tareas.

\section{Conclusión}

Para asegurar la correcta implementación de estos métodos y esta gestión es fundamental comenzar a trabajar desde las áreas de comunicación dando a conocer más aspectos generales de la planificación hacia el resto de la institución y buscar asegurar los mensajes que se emiten desde la organización especialmente, a través de lugares y personas menos convencionales y relacionadas con el proceso de comunicación formal de la institución.

Del mismo modo, explica Claus Møller (Barlow y Moller, 1996, 124) cómo se debe aprovechar cada oportunidad que se tenga de entrar en contacto con sus públicos y así obtener información de primera mano sobre sus percepciones y por supuesto, tomar acciones sobre ellas. Sin dejar de lado la oportunidad emergente de seguir en contacto y de ese modo testear los procesos de atención y, lo que es más importante, aprender de los propios errores.

Una solución posible es la incorporación de un mapa de contactos basado en los "momentos de la verdad" que tiene la organización como herramienta de comunicación. En este mapa se listará qué nivel de contacto tiene cada público y 
la descripción de cada tipo de interacción, la relación y el área de contacto con la organización. Mediante esta herramienta no se pretende controlar y dominar el proceso de la comunicación sino optimizar el flujo de información, desterrando los rumores y las confusiones que pudieran existir. De ningún modo pretende ser ni un método punitivo ni una herramienta de sometimiento, dado que, si fuera utilizada de esta manera, el resultado podría tornarse opuesto al deseado.

Este aprendizaje ayudará a interiorizar cuáles son las demandas de los públicos a través del conocimiento de su conjunto de expectativas y así poder proceder de acuerdo para presentar una propuesta de valor en sintonía con los públicos.

No obstante, para llevarlo a cabo será necesario comprender todo el proceso relacional. Tanto en los casos que se ha descrito anteriormente como "de hecho", dado su carácter equivalente con la imagen y su valor emotivo en los lazos con la marca o la organización; y en su carácter relacional creado, es decir, desarrollado estratégicamente desde la institución. Asimismo, en el proceso de anclaje de la relación con cada público debe hacerse foco en los intereses (Fisher y Ury, 1991, 40), tanto los propios como los de los públicos y dejar de lado las posiciones irreductibles que restan posibilidades de presentar propuestas de valor que sirvan para delinear relaciones mejores y más duraderas.

\section{Bibliografía}

Apfeldorfer, G. (2005). Las relaciones duraderas, amorosas, de amistad y profesionales. Barcelona: Paidóslbérica.

Barlow, J., \& Møller, C. (1996). A complaints a gift: Using customer feedback as a strategic tool. San Francisco: Berrett- Koehler Publishers.

Carlzon, J. (1991). El momento de la verdad. Madrid, España: Ediciones Díaz de Santos.

Cornelius, H., \& Faire, S. (1996). Tu ganas, yo gano, todos podemos ganar: Cómo resolver conflictos creativamente. Madrid: Gaia Ediciones. 
Dawkins, R. (1989). The selfish gene. Oxford: Oxford University Press.

Fisher, R., Ury, W., \&Patton, B. (1991). Getting to yes: Negotiating agreement without giving in. New York, N.Y: Penguin Books.

Fombrun, C. J. (1996). Reputation: Realizing value from the corporate image. Boston, Mass: Harvard Business School Press.

Horowitz, Peter y David. (1976) The Rockefellers, New York: Holt, Rinehart and Winston.

Manucci, M. (2006). La estrategia de los cuatro círculos: Diseñar el futuro en la incertidumbre del presente. Bogotá: Grupo Editorial Norma.

Rey Lennon, F. (2006) Edward Bernays. El día en que se inventaron las Relaciones Públicas. EDUCA.

Scheinsohn, D. (2000). Más allá de la imagen corporativa: Como crear valor a través de la comunicación estratégica. Buenos Aires, Argentina: Ediciones Macchi.

\section{Publicaciones Académicas}

Kent, Michael; Taylor, Maureen. "Toward a dialogic theory of Public Relations", diciembre de 2001, en Public Relations Review 28, 2002

Selzer, Trent. "Measuring the Impact of Public Relations: Using a CoorientationalApproachtoAnalyzetheOrganization-Publicrelationship"

Submitted to the Institute for Public Relations for the 2005 Ketchum Excellence in Public Relations Research Award

Para citar este artículo:

Ortner, Maximiliano (15-12-2011). LA RELACIÓN Y SU FUNCIÓN COMO MODELADORA DE LA IMAGEN.

REDMARKA - CIECID - Unidad de Investigación en Marketing Aplicado-Universidad de A Coruña.

Año IV, Número 7, V1, pp.61-76 ISSN 1852-2300

URL del Documento: cienciared.com.ar/ra/doc.php? $n=1596$

URL de la Revista: cienciared.com.ar/ra/revista.php?wid=39

REDMARKA UIMA-Universidad de A Coruña - CIECID 